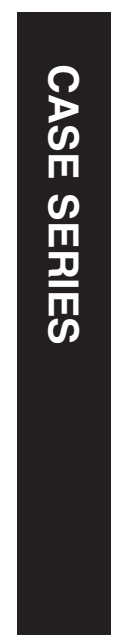

\title{
Optical coherence tomographic angiography shows reduced deep capillary flow in paracentral acute middle maculopathy
}

\begin{abstract}
Introduction Paracentral acute middle maculopathy (PAMM) has been described as an ischemic lesion of the middle retinal layers with a characteristic lamellar hyperreflective placoid appearance in the acute phase and thinning of the involved retinal layers in the chronic phase. Optical coherence tomographic angiography (OCTA) is a novel and non-invasive technique for imaging retinal capillary vasculature with en face segmentation capabilities.

Method Case series. We describe two patients with PAMM who underwent clinical examination and multimodal imaging including OCTA.

Results In the first patient, who presented with PAMM secondary to acute cilioretinal artery occlusion, OCTA demonstrated reduction in flow in the deep capillary plexus (DCP). One month later, OCTA revealed a flow void due to thinning of the GCL, INL, and OPL and paradoxical apparent ONL thickening. Similar findings of focal retinal lamellar ectopia were seen in the second patient, who had an incidentally detected chronic PAMM lesion.

Conclusions OCTA images the superficial and deep capillary plexi independently. PAMM is characterized by acute and chronic attenuation of the DCP flow signature. Focal lamellar ectopia in PAMM is discussed. Eye (2015) 29, 1620-1624; doi:10.1038/eye.2015.180; published online 18 September 2015
\end{abstract}

\section{Introduction}

Paracentral acute middle maculopathy (PAMM) was originally described by Sarraf et $a l^{1}$ as a
KK Dansingani ${ }^{1,2,3}, \mathrm{M}$ Inoue ${ }^{1,2}, \mathrm{M}$ Engelbert ${ }^{1,2,4}$ and KB Freund ${ }^{1,2,4,5}$ variant of AMN characterized by a hyperreflective parafoveal band on spectral domain optical coherence tomography (SD-OCT) involving the inner plexiform (IPL), outer plexiform (OPL), and inner nuclear layers (INL). $\mathrm{Yu}$ et al reported similar findings in a variety of retinal entities and hypothesized that ischemia of the intermediate and deep capillary plexus (DCP) was accountable.

Although difficult to detect clinically or with fluorescein angiography (FA), PAMM lesions are seen readily with near-infrared reflectance (nIR). Following resolution of the acute lesion, SD-OCT shows persistent thinning of the middle retinal layers.

Optical coherence tomographic angiography (OCTA) quantifies time-related differences in erythrocyte reflectivity to render flow maps of the retinal circulation with segmentation capabilities not afforded by dye-based angiography. ${ }^{3,4}$ We describe two patients with PAMM secondary to cilioretinal artery occlusion using multimodal imaging, including OCTA. Instruments used included the TRC-50XF fundus camera (Topcon Medical Systems, Paramus, NJ, USA), Heidelberg Spectralis (Heidelberg Engineering, Germany) and RTVue XR Avanti (Optovue, Fremont, CA, USA).

\section{Case report 1}

Figure 1 shows a 72-year-old male who presented with a 24-h history of a paracentral scotoma in the right eye. He had an episode of giant cell arteritis without visual complications 17 years previously. Visual acuities were $20 / 25$ (right eye) and $20 / 40$ (left eye). 


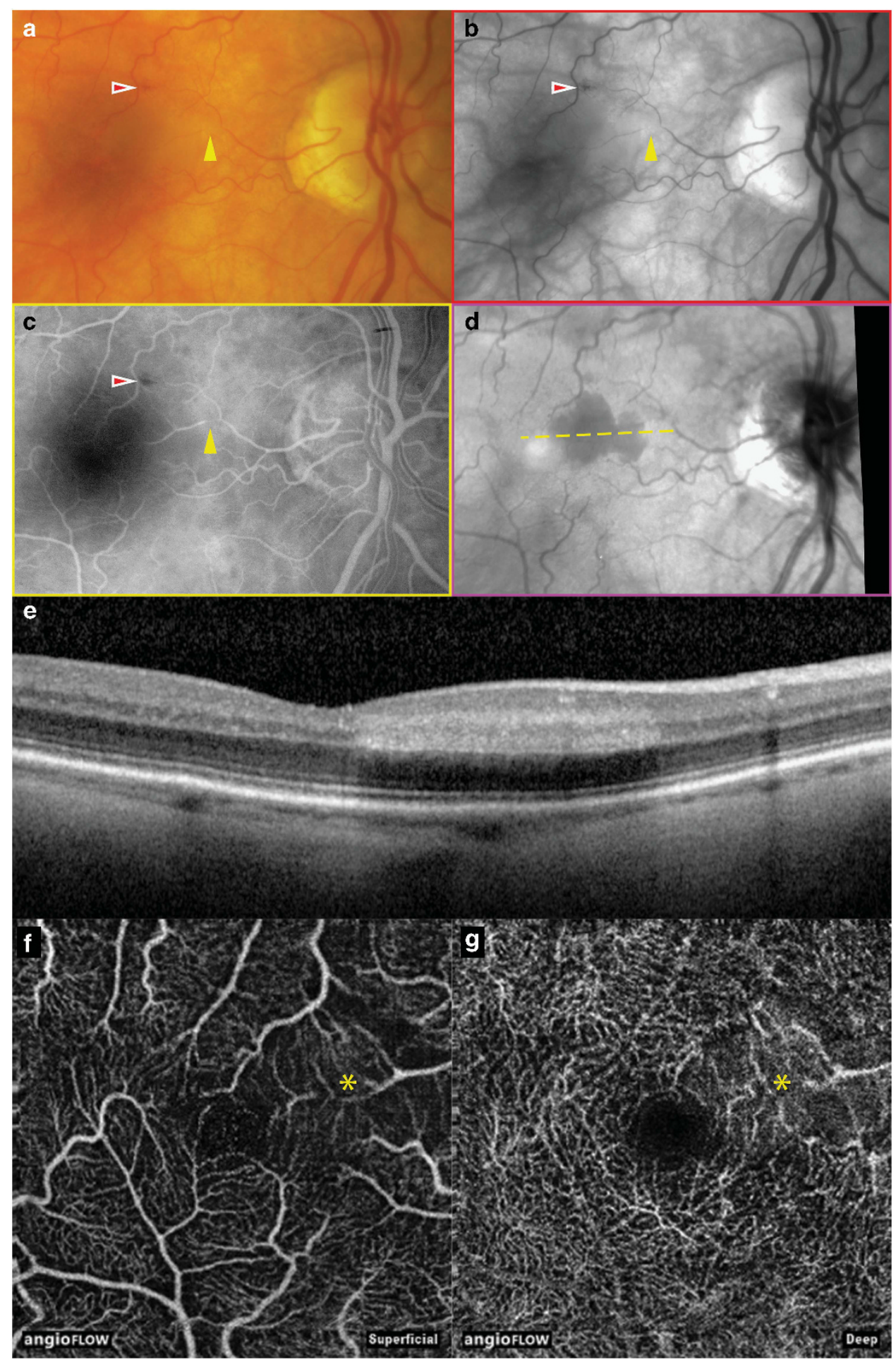

Figure 1 Multimodal imaging of the right eye of patient 1 presenting with acute PAMM. (a) Color photograph shows an intraretinal hemorrhage (red arrowhead) and a focal attenuation of a second order branch of a cilioretinal artery (yellow arrowhead), more evident in (b) red-free imaging and in (c) arterial phase fluorescein angiography. (d) nIR shows a hyporeflective lesion in the territory distal to the site of arteriolar occlusion. (e) Horizontal spectral domain optical coherence tomography (OCT) line scan through the lesion shows a band of increased intraretinal hyperreflectivity involving the IP, OP, and INL. (f-g) En face OCTA of the superficial and deep capillary plexi $(3 \times 3 \mathrm{~mm}$ scans), respectively, show reduced parafoveal flow that is more evident in the deep plexus (asterisks). 


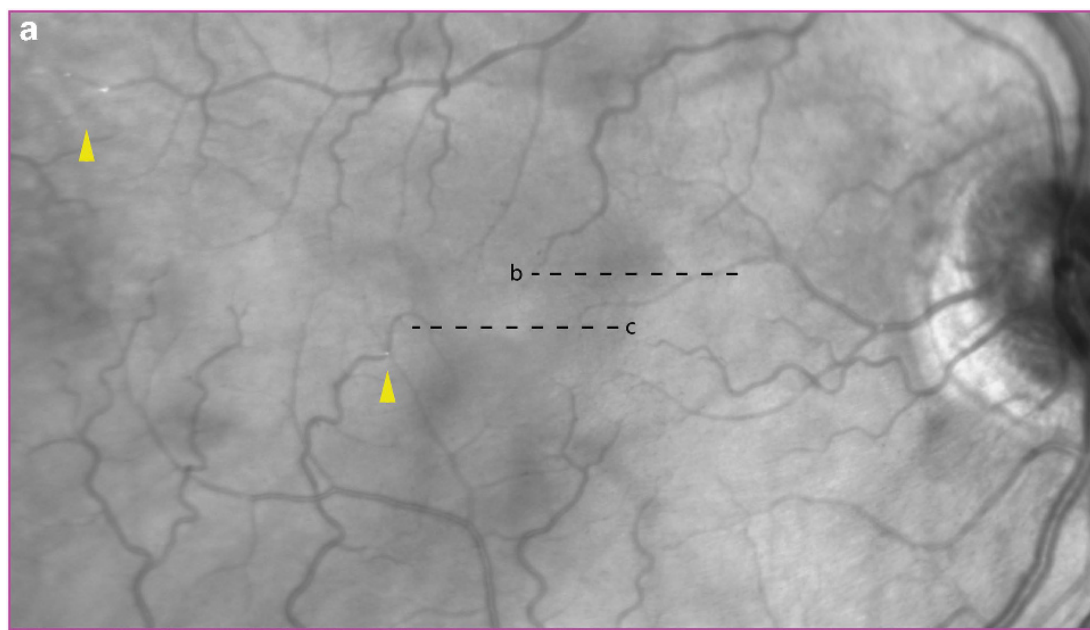

b

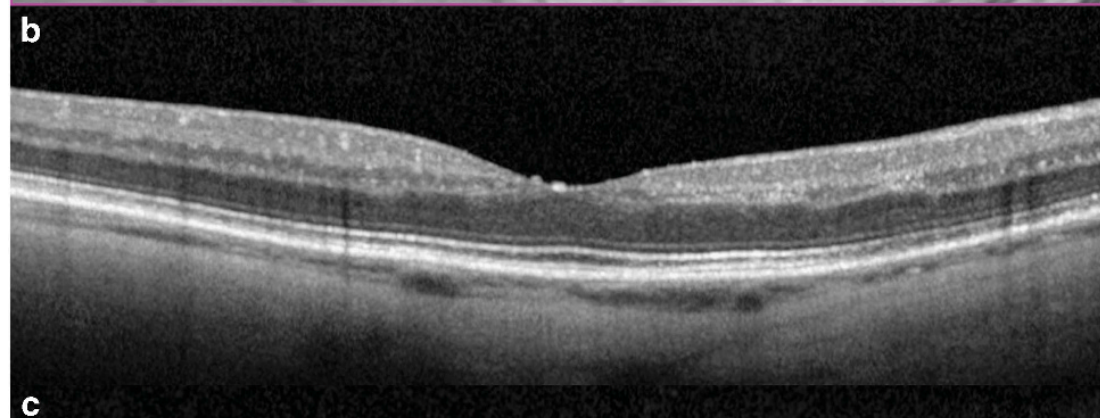

C
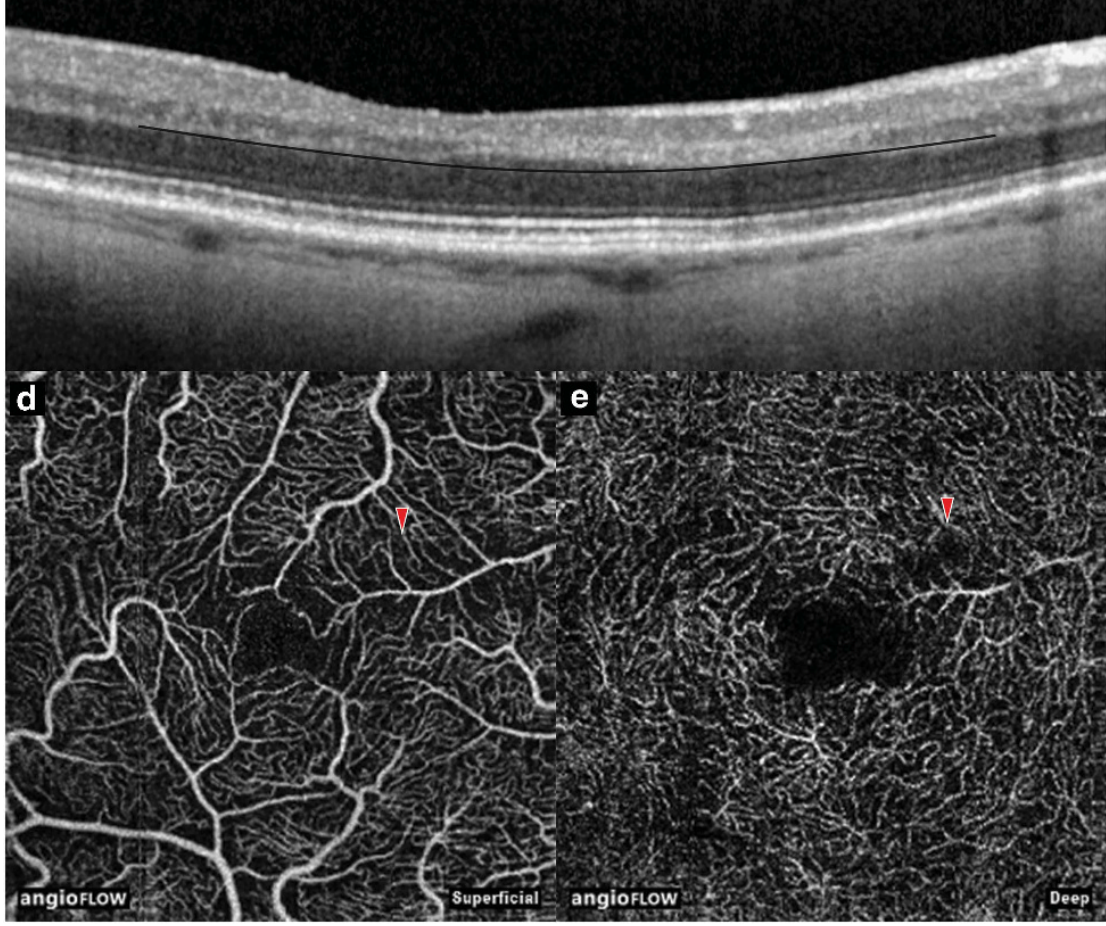

Figure 2 Selected multimodal imaging of the right eye of patient 1, one month after baseline presentation. (a) nIR shows apparent resolution of the hyporeflective paracentral lesion and reveals multiple emboli (arrowheads). (b-c) Horizontal spectral domain optical coherence tomography line scans through the fovea and PAMM lesion, respectively. The movable automated en face segmentation contour is described by a black curve overlaid at the outer border of the outer plexiform layer; alterations in retinal lamellar structure due to the PAMM lesion are discussed in the main text. (d) En face OCTA $(3 \times 3 \mathrm{~mm})$ shows a mild reduction in flow in the SCP at the site of the PAMM lesion (red arrowhead). (e) En face OCTA of the deep capillary plexus shows a parafoveal flow void (red arrowhead) which is discussed in the main text. 


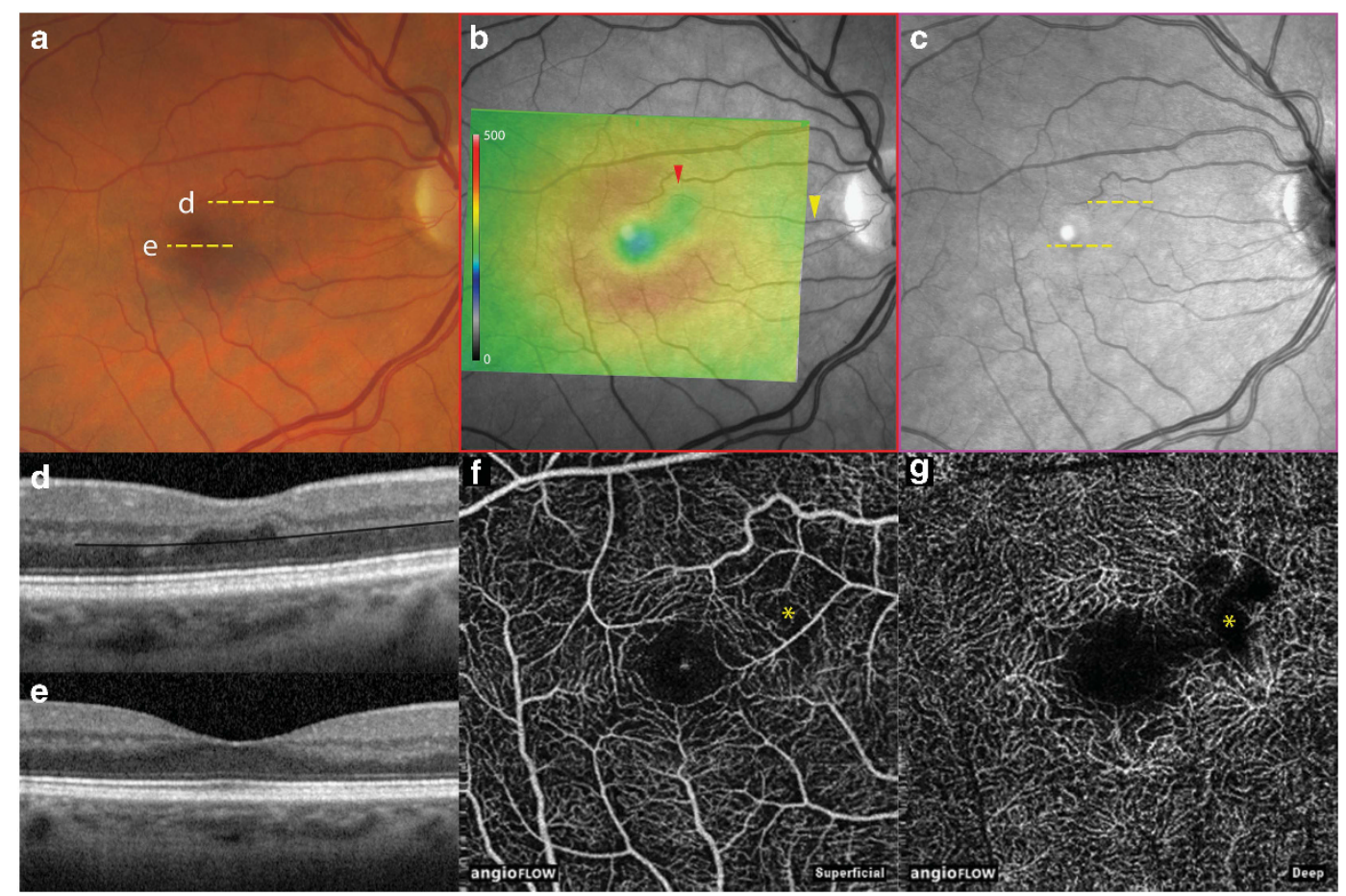

Figure 3 Multimodal imaging of the asymptomatic right eye of patient 2 shows findings consistent with a previous PAMM. (a) Color photograph appears to show normal retinal findings. (b) SD-OCT retinal thickness map (microns) overlaid onto a red-free photograph shows an area of retinal thinning (red arrowhead) that corresponds to the territory supplied by a second order cilioretinal arteriolar branch (yellow arrowhead). (c) Unremarkable nIR. (d) Horizontal SD-OCT line scan shows thinning of the IP, OP, and ONL, accounting for the focal area of reduced retinal thickness; apparent thickening of the ONL is also seen. The moveable automated en face segmentation contour is described by a black curve overlaid at the outer border of the OP layer, as in Figure 2c. (e) Horizontal SD-OCT line scan through the fovea shows normal retinal anatomy. (f) En face OCTA $(3 \times 3 \mathrm{~mm})$ of the SCP shows a mild reduction in flow in the area of the PAMM lesion (asterisk). (g) En face OCTA of the deep capillary plexus shows a parafoveal flow void (asterisk) which is discussed in the main text.

Ophthalmoscopy showed focal attenuation of a second order cilioretinal arteriolar branch in the right eye with a downstream area of reduced retinal transparency abutting the fovea. This area appeared hyporeflective on nIR, and SD-OCT showed a hyper-reflective PAMM lesion involving the IPL, OPL, and INL. Although FA appeared to show normal perfusion distal to the stenosis, OCTA documented reduced flow in the DCP.

One month later (Figure 2), the PAMM lesion was no longer visible on nIR but multiple new Hollenhorst plaques had appeared. Spectral domain OCT through the PAMM lesion showed thinning of the ganglion cell layer (GCL), IPL, and INL. The outer nuclear layer (ONL) appeared thickened with inward displacement and compression of the OPL and INL (lamellar ectopia).

The superficial capillary plexus (SCP) appeared unremarkable on OCTA. Automated segmentation of the DCP showed it to be a separate network with a morphology distinct from the SCP in most parts of the image. A flow void occurred where focal lamellar ectopia had shifted the OPL to exclude it from the en face segmentation boundary. Inward adjustment of the segmentation boundary did not show a DCP that was morphologically distinguishable from the SCP within the PAMM lesion.

\section{Case report 2}

Figure 3 shows a 57-year-old female seen for follow-up regarding a compensated branch retinal vein occlusion in her left eye. Visual acuities were $20 / 20$ (right eye) and $20 / 20$ (left eye). In the asymptomatic right eye, SD-OCT identified a focus of retinal thinning with INL attenuation following the distribution of a second order cilioretinal arteriolar branch. Lamellar ectopia was noted with marked apparent ONL thickening, shifting the DCP to exclude it from the en face segmentation zone. OCTA showed reduced flow in the SCP with a deeper flow void corresponding to the focal lamellar ectopia. Inward adjustment of the segmentation plane did not reveal a DCP flow signature that could be distinguished from the SCP. 


\section{Discussion}

The arrangement of retinal capillaries into superficial, intermediate, and deep plexi is known from histological and confocal imaging studies. ${ }^{5-7}$ Due to close proximity, OCTA segments of the SCP include flow signals from the nerve fiber layer and GCL; segments of the DCP include signals from the intermediate plexus. OCTA distinguishes the SCP from the DCP, where the depth resolution of FA is limited by vertical summation. The addition of OCTA to conventional multimodal imaging in acute and convalescent PAMM demonstrates reduced or absent DCP flow, supporting the view that PAMM is an ischemic lesion of the DCP.

Focal lamellar ectopia, as described here, is not well documented or addressed in the literature but its appreciation is critical for correct interpretation of OCTA, particularly since software tools for manually reshaping segmentation surfaces have not yet matured. It remains unclear whether lamellar ectopia results primarily from focal ONL thickening or whether this thickening is secondary to inward micro-forces produced by a contracting middle retinal lesion. Although apparent thickening of the ONL might actually represent an artifactual change in reflectivity, as suggested previously, ${ }^{1}$ rather than tissue ectopia, the inward change in contour of the INL and Henle fiber layer at the edges of the chronic PAMM lesions argues against this. Histopathological clarification may be impeded by tissue processing artifacts, unless specific staining identifies contracted glial tissue within chronic PAMM lesions.

Our observations support current hypotheses regarding PAMM as a sign of ischemic microvasculopathy and raise new questions as to how PAMM lesions may alter retinal lamellar architecture permanently.

\section{Summary}

What was known before

- Paracentral acute middle maculopathy (PAMM) is believed to be an ischemic lesion of the deep capillary plexus with a growing differential diagnosis. Optical coherence tomographic angiography (OCTA) resolves the superficial and deep capillary plexi independently.

What this study adds

- OCTA reveals reduced flow in the deep capillary plexus in acute and chronic PAMM, but correct interpretation of OCTA requires an appreciation of lamellar tissue ectopia seen in convalescent PAMM lesions. These architectural changes have not been addressed fully in previous literature.

\section{Conflict of interest}

$\mathrm{KBF}$ is a consultant for the following companies: Genentech, Optos, ThromboGenics, Ohr Pharmaceutical, Bayer HealthCare and Heidelberg Engineering. ME was former consultant for Bayer and Genentech.

\section{Acknowledgements}

This study has received financial support from: LuEsther T. Mertz Retinal Research Center, Manhattan Eye, Ear and Throat Hospital, New York and the Macula Foundation, New York, NY, USA.

\section{Disclaimer}

This work is original. The abstract has been accepted for the ARVO 2015 Imaging in the Eye Symposium, but this work has not been published elsewhere. The funding organizations had no role in the design or conduct of this research.

\section{References}

1 Sarraf D, Rahimy E, Fawzi AA, Sohn E, Barbazetto I, Zacks DN et al. Paracentral acute middle maculopathy: a new variant of acute macular neuroretinopathy associated with retinal capillary ischemia. JAMA Ophthalmol 2013; 131(10): 1275-1287.

2 Yu S, Pang CE, Gong Y, Freund KB, Yannuzzi LA, Rahimy E et al. The spectrum of superficial and deep capillary ischemia in retinal artery occlusion. Am J Ophthalmol 2015; 159(1): 53-63 e51-52.

3 Jia Y, Tan O, Tokayer J, Potsaid B, Wang Y, Liu JJ et al. Split-spectrum amplitude-decorrelation angiography with optical coherence tomography. Opt Express 2012; 20(4): 4710-4725.

4 Nagiel A, Sadda SR, Sarraf D. A promising future for optical coherence tomography angiography. JAMA Ophthalmol 2015; 133(6): 629-630.

5 Dorrell MI, Friedlander M, Smith LEH. Retinal Vascular Development. In: Joussen AM, Gardner TW, Kirchhof B, Ryan SJ (eds). Retinal Vascular Disease. Springer-Verlag: Berlin, Heidelberg, NY, USA, 2007, pp 29-30.

6 Foreman DM, Bagley S, Moore J, Ireland GW, McLeod D, Boulton ME. Three dimensional analysis of the retinal vasculature using immunofluorescent staining and confocal laser scanning microscopy. Br J Ophthalmol 1996; 80(3): 246-251.

7 Tan PE, Yu PK, Balaratnasingam C, Cringle SJ, Morgan WH, McAllister IL et al. Quantitative confocal imaging of the retinal microvasculature in the human retina. Invest Ophthalmol Vis Sci 2012; 53(9): 5728-5736. 